\title{
On the Boussinesq equations with anisotropic filter in a vertical pipe
}

\author{
Luigi C. Berselli* \\ Dipartimento di Matematica, \\ Università di Pisa, \\ Via F. Buonarroti 1/c, 56125 Pisa, ITALY \\ berselli@dma.unipi.it \\ Davide Catania \\ DICATAM, Sezione Matematica, \\ Università di Brescia, \\ Via Valotti 9, 25133 Brescia, ITALY \\ davide.catania@unibs.it
}

December 3, 2014

\begin{abstract}
We propose a new Large Eddy Simulation (LES) model for the Boussinesq equations. We consider the motion in a three-dimensional domain with solid walls, and in a particular geometrical situation we look for solutions which are periodic in the vertical direction and satisfy homogeneous Dirichlet conditions on the lateral boundary. We are thus modeling a vertical pipe and one main difficulty is that of considering regularizations of the equation which are well behaved also in presence of a boundary. The LES model we consider is then obtained by introducing a vertical filter, which is the natural one for the setting that we are considering. The related interior closure problem is treated in a standard way with a simplified-Bardina deconvolution model. The most technical analytical point is related to the fact that anisotropic filters provide less regularity than the isotropic ones and, in principle, the density term appearing in the Boussinesq equations may behave very differently from the velocity. We are able to define an appropriate notion of regular weak solution, for which we prove existence, uniqueness, and we also show that the energy associated to the model is exactly preserved.
\end{abstract}

Keywords. Boussinesq equations, anisotropic vertical filter, alpha-models, turbulent flow in domain with boundary, Large Eddy Simulation (LES), approximate deconvolution methods.

\footnotetext{
*Both authors are members of the Gruppo Nazionale per l'Analisi Matematica, la Probabilità e le loro Applicazioni (GNAMPA) of the Istituto Nazionale di Alta Matematica (INdAM). The first author is member of the National Project (PRIN 2012L5WXHJ_004) Nonlinear Hyperbolic Partial Differential Equations, Dispersive and Transport Equations: theoretical and applicative aspects.
} 
MSC: 76D05; 35Q30, 76F65, 76D03.

\section{Introduction}

We are interested in developing and studying a Large Eddy Simulation Model (LES) useful to simulate some mixing phenomena for turbulent flows, when direct numerical simulations are not feasible, because computationally too expensive. Since the effects of boundaries are of fundamental importance for formation and development of turbulence and mixing, we are mainly focused to find a suitable LES model which can be successfully applied also in presence of solid boundaries. In particular, we will consider the Boussinesq equations in a vertical cylindrical domain, with a filtering which is weaker than the usual one.

The LES is achieved by applying a suitable filter, which neglects the small-scale motions, makes the solutions smoother, and allows numerical simulations. In particular, we consider a special class of differential filters. A general overview on LES models is given in [6]. Moreover, LES models are characterized by a deterministic behavior, while pointwise (unfiltered) turbulent flows manifest randomness. A successful example of regularized models is given by $\alpha$-models (see the introductions in $[10,11,15,18,22])$, where filtering is obtained through the inverse of the Helmholtz operator $A=I-\alpha^{2} \Delta$, where $\Delta$ is the Laplace operator, and $\alpha>0$ represents the filtering spatial scale. However, the presence of the boundary imposes boundary conditions on the filter, so that the usual isotropic filters are unsuited to be applied due to the lack of commutation between filtering and differentiation (see [6, Ch. 9]), which yields problems in deriving the filtered equations. To overcome this difficulty, anisotropic filters have been already proposed for numerical simulations in Deardorff [14] and, even for differential filters, in Germano [17], where it is pointed out that anisotropic filters are less memory consuming than their isotropic versions.

A first example of anisotropic regularization in the context of $\alpha$-models (see also the earlier work with discrete methods [23]) can be found in [3], where the horizontal filter determined by the inverse of $A_{\mathrm{h}}=I-\alpha^{2} \Delta_{\mathrm{h}}$ (where $\Delta_{\mathrm{h}}:=\partial_{x_{1}}^{2}+\partial_{x_{2}}^{2}$ is the Laplacian in the horizontal variables) replaces the usual Helmholtz one with the full Laplacian. The idea of using this filtering comes also from the previous analytical results in [13] and in [3], where the anisotropic filter is applied to the incompressible Navier-Stokes equations in a 3D bounded channel domain, with periodic horizontal boundary conditions and homogeneous Dirichlet conditions on the upper and lower boundaries. Existence and uniqueness of so-called regular weak solutions are then proved. In [4] the horizontal filter has been applied in several ways to the Oberbeck-Boussinesq equations (or simply Boussinesq equations) and existence and uniqueness (in one case) of solutions have been obtained for the corresponding LES model, thus continuing the study of LES models applied to stratified flows (see [19] and the references therein for numerical results in this direction). As explained in [4], the Boussinesq equations are well suited for the simulation of some mixing phenomena involved in certain geophysical and oceanic flows. They couple the Navier-Stokes equations for the fluid velocity (whose difficulties are shared) with a transport equation (requiring an additional analysis) for a scalar, usually interpreted as a density or temperature perturbation.

An analogous result has been obtained by Ali [1] in a vertical cylindrical domain for the NavierStokes equations regularized through the inverse of the differential operator $A_{3}=I-\alpha^{2} \partial_{3}^{2}$, where $\partial_{3}$ is the derivative with respect to the vertical component. Here, we further develop ideas coming from $[1,2]$ and we consider the LES model obtained by filtering the Boussinesq equations in a vertical cylindrical domain, i.e., a bounded domain periodic in the vertical direction endowed with homogeneous Dirichlet conditions on the lateral boundary, and through the vertical filter given by 
$A_{3}^{-1}$. In particular we find a suitable setting to handle also the density equation; together with existence and uniqueness of regular weak solutions, we also prove that the energy of the model is exactly preserved. In our analysis, we consider a zeroth-order deconvolution model à la Stolz and Adams (or simplified-Bardina), see [9, 18]. We refer to $[1,4,7,8]$ for results concerning Approximate Deconvolution Models (ADM) with general order of deconvolution for the Navier-Stokes equations and the Boussinesq equations. We do not enter the game of considering fractional variants of the filter (anyway a fractional power of the Laplacian larger than one-half will suffice for most results), in order to find the critical spaces and so on, since we are mainly interested to propose an easy applicable method, which can be used and implemented in an efficient way also by practitioners.

\subsection{Plan of the paper}

Section 2 includes the main notations and the functional spaces we will use. In Section 3 we introduce the LES model. The main result and its proof are detailed in four subsections: Preliminary results; Existence and energy inequality; Energy identity; Uniqueness and continuous dependence on the data.

\section{Notations}

The Boussinesq Equations in non-dimensional form read as follows:

$$
\begin{array}{ll}
\partial_{t} \boldsymbol{u}+\nabla \cdot(\boldsymbol{u} \otimes \boldsymbol{u})-\nu \Delta \boldsymbol{u}+\nabla \pi=-\vartheta e_{3} & (t, x) \in(0, T) \times D, \\
\nabla \cdot \boldsymbol{u}=0 & (t, x) \in(0, T) \times D, \\
\partial_{t} \vartheta+\nabla \cdot(\vartheta \boldsymbol{u})-K \Delta \vartheta=0 & (t, x) \in(0, T) \times D,
\end{array}
$$

where the unknowns are the velocity vector field $\boldsymbol{u}$, and the scalar fields $\pi$ and $\vartheta$, where $\pi$ is the pressure, while $\vartheta$ is generally interpreted as a density or as a temperature fluctuation. The positive constants $\nu$ and $K$ are known respectively as kinematic viscosity and diffusivity, while $e_{3}:=(0,0,1)$.

Due to the particular geometry in which we will consider the problem, and since the proofs will exploit such a special form, we use the following notation

$$
\boldsymbol{x}=\left(x_{1}, x_{2}, x_{3}\right) \quad \text { and } \quad \boldsymbol{x}_{\mathrm{h}}=\left(x_{1}, x_{2}\right),
$$

where "h" stays for "horizontal". For a vector function $\boldsymbol{w}=\left(w^{1}, w^{2}, w^{3}\right)$, we set $\boldsymbol{w}^{\mathrm{h}}=\left(w^{1}, w^{2}\right)$ in such a way that $\boldsymbol{w}=\left(w^{\mathrm{h}}, w^{3}\right)$. We also use the same splitting on differential operators and if $\partial_{j}=\partial_{x_{j}}$, then

$$
\nabla_{\mathrm{h}}=\left(\partial_{1}, \partial_{2}\right) \text { and } \quad \nabla=\left(\nabla_{\mathrm{h}}, \partial_{3}\right) .
$$

We denote by $\Omega \subset \mathbb{R}^{2}$ a smooth bounded open domain and the space domain is

$$
D=\Omega \times]-\pi, \pi\left[=\left\{\boldsymbol{x} \in \mathbb{R}^{3}:\left(x_{1}, x_{2}\right) \in \Omega,-\pi<x_{3}<\pi\right\},\right.
$$

with $2 \pi$-periodicity with respect to $x_{3}$ and homogeneous Dirichlet boundary conditions on $\Gamma=$ $\partial \Omega \times]-\pi, \pi[$. 
We introduce the following function spaces, which are natural with the above splitting of variables:

$$
\begin{aligned}
& \mathrm{L}^{2}(D)=\left\{\phi: D \rightarrow \mathbb{R} \text { measurable, } 2 \pi \text {-periodic in } x_{3}, \int_{D}|\phi|^{2} \mathrm{~d} \boldsymbol{x}<+\infty\right\}, \\
& \mathrm{L}_{0}^{2}(D)=\left\{\phi \in \mathrm{L}^{2}(D), \int_{-\pi}^{\pi} \phi \mathrm{d} x_{3}=0\right\}, \\
& \boldsymbol{H}=\left\{\boldsymbol{\phi} \in \mathbf{L}_{0}^{2}(D): \nabla \cdot \boldsymbol{\phi}=0 \text { in } D, \boldsymbol{\phi} \cdot \boldsymbol{n}=0 \text { on } \Gamma\right\}
\end{aligned}
$$

( $\boldsymbol{n}$ is the outward unit normal vector on $\Gamma$ ), all with $\mathrm{L}^{2}$ norm denoted by $\|\cdot\|$, and scalar product $(\cdot, \cdot)$ in $\mathrm{L}^{2}$. Here and in the following, we use boldface letters to denote spaces of vector and or tensor valued functions (with respect to the space variables). A delicate interplay is that between horizontal $\left(x_{\mathrm{h}}\right)$ and vertical (namely $x_{3}$ ) variables, so we define the following functional spaces:

$$
\begin{aligned}
& H_{\text {vert }}^{1}=\left\{\phi \in \mathrm{L}_{0}^{2}(D): \partial_{3} \phi \in \mathrm{L}^{2}(D)\right\}, \\
& \boldsymbol{V}_{\text {vert }}=\left\{\boldsymbol{\phi} \in \boldsymbol{H}: \partial_{3} \phi \in \mathbf{L}^{2}(D)\right\}, \\
& \boldsymbol{V}=\left\{\boldsymbol{\phi} \in \boldsymbol{H}: \nabla \boldsymbol{\phi} \in \mathbf{L}^{2}(D) \text { and } \boldsymbol{\phi}=\mathbf{0} \text { on } \Gamma\right\}, \\
& H_{\text {vert }}^{2}=\left\{\phi \in H_{\text {vert }}^{1}: \partial_{3} \nabla \phi \in \mathbf{L}^{2}(D)\right\},
\end{aligned}
$$

and denote by $\boldsymbol{V}^{*}$ the dual space to $\boldsymbol{V}$. We denote by $\mathrm{L}^{p}$ (with norm $\|\cdot\|_{\mathrm{L}^{p}}$ ) and $\mathrm{H}^{m}$ (with norm $\|\cdot\|_{\mathrm{H}^{m}}$ ) classical Lebesgue and Sobolev spaces. Weakly continuous functions $[0, T] \mapsto X$ are denoted by the symbol $\mathscr{C}_{\mathrm{w}}(0, T ; X)$.

\section{On the LES model}

We introduce the vertical ( $x_{3}$ direction) filter by means of the operator $A_{3}=I-\alpha^{2} \partial_{3}^{2}$ as follows:

$$
\overline{\boldsymbol{v}}=A_{3}^{-1} \boldsymbol{v},
$$

where both $\overline{\boldsymbol{v}}$ and $\boldsymbol{v}$ are periodic in $x_{3}$ and with vanishing mean value with respect to $x_{3}$. By filtering the equations (and exploiting the commutation of filter and derivatives with respect to $x_{3}$, due to periodicity), we obtain

$$
\begin{array}{ll}
\partial_{t} \overline{\boldsymbol{u}}+\nabla \cdot \overline{(\boldsymbol{u} \otimes \boldsymbol{u})}-\nu \Delta \overline{\boldsymbol{u}}+\nabla \bar{\pi}=-\bar{\vartheta} e_{3} & (t, x) \in(0, T) \times D, \\
\nabla \cdot \overline{\boldsymbol{u}}=0 & (t, x) \in(0, T) \times D, \\
\partial_{t} \bar{\vartheta}+\nabla \cdot \overline{(\vartheta \boldsymbol{u})}-K \Delta \bar{\vartheta}=0 & (t, x) \in(0, T) \times D .
\end{array}
$$

We set $\boldsymbol{w}=\overline{\boldsymbol{u}}, q=\bar{\pi}, \rho=\bar{\vartheta}$, so that $\boldsymbol{u}=A_{3} \boldsymbol{w}$; we solve the interior closure problem by the approximations

$$
\begin{aligned}
& \text { a) } \overline{\overline{\boldsymbol{u} \otimes \boldsymbol{u}}} \approx \overline{\overline{\boldsymbol{u}} \otimes \overline{\boldsymbol{u}}}=\overline{\boldsymbol{w} \otimes \boldsymbol{w}}, \\
& \text { b) } \overline{\vartheta \boldsymbol{u}} \approx \overline{\bar{\vartheta}} \overline{\boldsymbol{u}}=\overline{\rho \boldsymbol{w}},
\end{aligned}
$$


which can be seen as a zeroth order deconvolution model $[7,9,18]$. We finally deduce the following system of partial differential equations, which depends only on "filtered" variables $(\boldsymbol{w}, q, \rho)$ :

$$
\begin{array}{lc}
\partial_{t} \boldsymbol{w}+\nabla \cdot \overline{(\boldsymbol{w} \otimes \boldsymbol{w})}-\nu \Delta \boldsymbol{w}+\nabla q=-\rho e_{3} & (t, x) \in(0, T) \times D, \\
\nabla \cdot \boldsymbol{w}=0 & (t, x) \in(0, T) \times D, \\
\partial_{t} \rho+\nabla \cdot(\overline{\rho \boldsymbol{w}})-K \Delta \rho=0 & (t, x) \in(0, T) \times D .
\end{array}
$$

Remark 3.1 We use the same filter for all the equations to simplify notation. However the proof still works by introducing two different filters, i.e. two scale lengths $\alpha_{1}$ and $\alpha_{2}$, for $\boldsymbol{u}$ and $\vartheta$ respectively (see [4]). For other related models with different filters similar to the Leray or simplifiedBardina, see also Ali and Ammari [2].

Definition (Regular weak solution) We say that $(\boldsymbol{w}, \rho)$ with $\boldsymbol{w}:(0, T) \times D \rightarrow \mathbb{R}^{3}$ and $\rho:(0, T) \times D \rightarrow$ $\mathbb{R}$ is a regular weak solution (omitting the pressure term $q$ ) to system (1)-(2)-(3), with $\boldsymbol{w}=\mathbf{0}$ and $\rho=0$ on $] 0, T\left[\times \Gamma\right.$, and $(\boldsymbol{w}(0, \boldsymbol{x}), \rho(0, \boldsymbol{x}))=\left(\boldsymbol{w}_{0}, \rho_{0}\right) \in \boldsymbol{V}_{\text {vert }} \times H_{\text {vert }}^{1}$, when the following properties are verified.

- Regularity:

$$
\begin{aligned}
& \boldsymbol{w} \in \mathrm{L}^{\infty}\left(0, T ; \boldsymbol{V}_{\text {vert }}\right) \cap \mathrm{L}^{2}\left(0, T ; \boldsymbol{V} \cap \boldsymbol{H}_{\text {vert }}^{2}\right) \cap \mathscr{C}_{\mathrm{w}}\left(0, T ; \boldsymbol{V}_{\text {vert }}\right), \\
& \partial_{t} \boldsymbol{w} \in \mathrm{L}^{2}\left(0, T ; \boldsymbol{V}^{*}\right), \\
& \rho \in \mathrm{L}^{\infty}\left(0, T ; H_{\text {vert }}^{1}\right) \cap \mathrm{L}^{2}\left(0, T ; \mathrm{H}^{1} \cap H_{\text {vert }}^{2}\right) \cap \mathscr{C}_{\mathrm{w}}\left(0, T ; H_{\text {vert }}^{1}\right), \\
& \partial_{t} \rho \in \mathrm{L}^{2}\left(0, T ; \mathrm{H}^{-1}\right) .
\end{aligned}
$$

- Weak formulation:

$$
\begin{aligned}
& \int_{0}^{+\infty}\left\{\left(\boldsymbol{w}, \partial_{t} \boldsymbol{\varphi}\right)-\nu(\nabla \boldsymbol{w}, \nabla \boldsymbol{\varphi})+(\boldsymbol{w} \otimes \boldsymbol{w}, \nabla \overline{\boldsymbol{\varphi}})\right\}(s) \mathrm{d} s \\
& \quad=\int_{0}^{+\infty}\left(\rho e_{3}, \boldsymbol{\varphi}\right)(s) \mathrm{d} s-(\boldsymbol{w}(0), \boldsymbol{\varphi}(0)) \\
& \int_{0}^{+\infty}\left\{\left(\rho, \partial_{t} \psi\right)+(\rho \boldsymbol{w}, \nabla \bar{\psi})-K(\nabla \rho, \nabla \psi)\right\}(s) \mathrm{d} s=-(\rho(0), \psi(0))
\end{aligned}
$$

for each $\varphi \in\left(\mathscr{C}_{0}^{\infty}\left([0, T[\times D))^{3}\right.\right.$ such that $\nabla \cdot \varphi=0$, and for each $\psi \in \mathscr{C}_{0}^{\infty}([0, T[\times D)$ or, equivalently (see Galdi [16]) if

$$
\begin{aligned}
& \int_{t_{0}}^{t_{1}}\left\{\left(\boldsymbol{w}, \partial_{t} \boldsymbol{\varphi}\right)-\nu(\nabla \boldsymbol{w}, \nabla \boldsymbol{\varphi})+(\boldsymbol{w} \otimes \boldsymbol{w}, \nabla \overline{\boldsymbol{\varphi}})\right\}(s) \mathrm{d} s \\
& \quad=\int_{t_{0}}^{t_{1}}\left(\rho e_{3}, \boldsymbol{\varphi}\right)(s) \mathrm{d} s+\left(\boldsymbol{w}\left(t_{1}\right), \boldsymbol{\varphi}\left(t_{1}\right)\right)-\left(\boldsymbol{w}\left(t_{0}\right), \boldsymbol{\varphi}\left(t_{0}\right)\right), \\
& \int_{t_{0}}^{t_{1}}\left\{\left(\rho, \partial_{t} \psi\right)+(\rho \boldsymbol{w}, \nabla \bar{\psi})-K(\nabla \rho, \nabla \psi)\right\}(s) \mathrm{d} s=\left(\rho\left(t_{1}\right), \psi\left(t_{1}\right)\right)-\left(\rho\left(t_{0}\right), \psi\left(t_{0}\right)\right)
\end{aligned}
$$

for each $0 \leq t_{0} \leq t_{1}<T$. 
Remark 3.2 Since $\boldsymbol{w} \in \mathrm{L}^{2}(0, T ; \boldsymbol{V})$ and $\partial_{t} \boldsymbol{w} \in \mathrm{L}^{2}\left(0, T ; \boldsymbol{V}^{*}\right)$, by classical interpolation arguments we can deduce $\boldsymbol{w} \in \mathscr{C}([0, T] ; \boldsymbol{H})$. Similarly, we obtain $\rho \in \mathscr{C}\left([0, T] ; \mathrm{L}_{0}^{2}(D)\right)$.

Moreover, since $\partial_{3} \boldsymbol{w} \in \mathrm{L}^{\infty}(0, T ; \boldsymbol{H}) \cap \mathrm{L}^{2}(0, T ; \boldsymbol{V})$ and $\nabla \cdot \boldsymbol{w}=0$, we have also

$$
\nabla_{\mathrm{h}} \cdot \boldsymbol{w}^{\mathrm{h}}=-\partial_{3} w^{3} \in \mathrm{L}^{\infty}\left(0, T ; \mathrm{L}^{2}\right) \cap \mathrm{L}^{2}\left(0, T ; \mathrm{H}^{1}\right) .
$$

The main result of the paper, which justifies the introduction of the LES model (1)-(2)-(3) (at least in a pipe) is Theorem 3.3, showing together with well-posedness also a good energy budget.

We will skip some details in the proof of the main results, since they follow the same lines of previous ones in $[1,3,7]$. Hence, we show the main steps needed to put the new system in a similar framework and - in addition - we focus more precisely on the role of the "energy" of the system.

Theorem 3.3 Let be given $\left(\boldsymbol{w}_{0}, \rho_{0}\right) \in \boldsymbol{V}_{\text {vert }} \times H_{\text {vert }}^{1}$ and $\alpha, \nu, K>0$. Then, there exists a unique regular weak solution to (1)-(2)-(3), with $\boldsymbol{w}=\mathbf{0}$ and $\rho=0$ on $] 0, T[\times \Gamma$, and $(\boldsymbol{w}(0, \boldsymbol{x}), \rho(0, \boldsymbol{x}))=$ $\left(\boldsymbol{w}_{0}, \rho_{0}\right)$. Moreover, this solution depends weakly continuously on the data and satisfies the energy (of the model) identity

$$
\begin{aligned}
& \frac{1}{2}\left(\|\boldsymbol{w}(t)\|^{2}+\alpha^{2}\left\|\partial_{3} \boldsymbol{w}(t)\right\|^{2}+\|\rho(t)\|^{2}+\alpha^{2}\left\|\partial_{3} \rho(t)\right\|^{2}\right)+\nu \int_{0}^{t}\left(\|\nabla \boldsymbol{w}(s)\|^{2}+\alpha^{2}\left\|\partial_{3} \nabla \boldsymbol{w}(s)\right\|^{2}\right) \mathrm{d} s \\
& \quad+K \int_{0}^{t}\left(\|\nabla \rho(s)\|^{2}+\alpha^{2}\left\|\partial_{3} \nabla \rho(s)\right\|^{2}\right) \mathrm{d} s \\
& =\frac{1}{2}\left(\|\boldsymbol{w}(0)\|^{2}+\alpha^{2}\left\|\partial_{3} \boldsymbol{w}(0)\right\|^{2}+\|\rho(0)\|^{2}+\alpha^{2}\left\|\partial_{3} \rho(0)\right\|^{2}\right)-\int_{0}^{t}\left(\rho(s) e_{3}, \boldsymbol{w}(s)\right) \mathrm{d} s .
\end{aligned}
$$

Clearly the same results hold also in the space periodic setting, where implementation of the differential filter is straightforward and all proofs are easier.

\subsection{Preliminary results}

A crucial result is the following inequality for the convective term, which involves the anisotropic derivative $\partial_{3} \nabla$ instead of the Laplace operator, and will be used very often in the following. It is taken from Ali [1].

Lemma 3.4 Let us assume that $\boldsymbol{u}, \boldsymbol{v}, \boldsymbol{w}$ are smooth enough vector fields such that $\boldsymbol{u}, \boldsymbol{w}$ satisfy homogeneous Dirichlet boundary conditions on $\Gamma$. Then, there exists a positive constant $C$ such that, for any real $s>1 / 2$, there holds

$$
|((\boldsymbol{u} \cdot \nabla) \boldsymbol{v}, \boldsymbol{w})| \leq C\|\boldsymbol{u}\|^{1 / 2}\|\nabla \boldsymbol{u}\|^{1 / 2}\|\boldsymbol{w}\|^{1 / 2}\|\nabla \boldsymbol{w}\|^{1 / 2}\|\nabla \boldsymbol{v}\|^{1-\frac{1}{2 s}}\left\|\partial_{3}^{s} \nabla \boldsymbol{v}\right\|^{\frac{1}{2 s}} .
$$

Sketch of the proof. First, we use the Hölder inequality with different exponents for the vertical variable and the horizontal variables (here, the subscripts "v" and "h" denote respectively the vertical and horizontal components):

$$
|((\boldsymbol{u} \cdot \nabla) \boldsymbol{v}, \boldsymbol{w})| \leq C\|\boldsymbol{u}\|_{\mathbf{L}_{\mathrm{v}}^{2} \mathbf{L}_{\mathrm{h}}^{4}}\|\nabla \boldsymbol{v}\|_{\mathbf{L}_{\mathrm{v}}^{\infty} \mathbf{L}_{\mathrm{h}}^{2}}\|\boldsymbol{w}\|_{\mathbf{L}_{\mathrm{v}}^{2} \mathbf{L}_{\mathrm{h}}^{4}} .
$$

The first and third term can be estimated by using the following inequality, proved by Paicu and Raugel [20]:

$$
\|\boldsymbol{u}\|_{\mathbf{L}_{\mathrm{v}}^{2} \mathbf{L}_{\mathrm{h}}^{4}} \leq C\|\boldsymbol{u}\|^{1 / 2}\left\|\nabla_{\mathrm{h}} \boldsymbol{u}\right\|^{1 / 2}
$$


Moreover, using Fourier expansions, it is possible to prove that $\|g\|_{\mathrm{L}^{\infty}} \leq C\|g\|^{1-\frac{1}{2 s}}\|g\|_{\mathrm{H}^{s}}^{\frac{1}{2 s}}$. From this inequality, we deduce

$$
\|\boldsymbol{u}\|_{\mathbf{L}_{\mathrm{v}}^{\infty} \mathbf{L}_{\mathrm{h}}^{2}} \leq C\|\boldsymbol{u}\|^{1-\frac{1}{2 s}}\left\|\partial_{3}^{s} \boldsymbol{u}\right\|^{\frac{1}{2 s}}
$$

(see Ali [1] for the details). By applying this result when $\boldsymbol{u}=\nabla \boldsymbol{v}$, we easily get the claim.

Let us note that, if $\boldsymbol{u}$ is divergence free, we have

$$
((\boldsymbol{u} \cdot \nabla) \boldsymbol{w}, \boldsymbol{v})=-((\boldsymbol{u} \cdot \nabla) \boldsymbol{v}, \boldsymbol{w}),
$$

and the roles of $\boldsymbol{v}$ and $\boldsymbol{w}$ in the previous lemma can be swapped, if now $\boldsymbol{v}$ is vanishing on $\Gamma$.

\subsection{Existence and energy inequality}

In order to construct regular weak solutions, we consider the Galerkin approximate solutions

$$
\boldsymbol{w}_{m}(t, \boldsymbol{x})=\sum_{j=1}^{m} g_{m}^{j}(t) \boldsymbol{E}_{j}(\boldsymbol{x}) \quad \text { and } \quad \rho_{m}(t, \boldsymbol{x})=\sum_{j=1}^{m} \gamma_{m}^{j}(t) E_{j}(\boldsymbol{x}),
$$

where $\boldsymbol{E}_{j}$ and $E_{j}$ are smooth eigenfunctions of the Stokes and Laplace operator on $D$, with homogeneous Dirichlet boundary conditions on $\Gamma$ and periodicity with respect to $x_{3}$ (see Rummler [21]). We will take advantage from using this special basis, since many calculations are then justified. If $\boldsymbol{P}_{m}$ denotes the projection on $\operatorname{span}\left\{\boldsymbol{E}_{1}, \ldots, \boldsymbol{E}_{m}\right\}$ and similarly for $P_{m}$, the couple $\left(\boldsymbol{w}_{m}, \rho_{m}\right)$ has to solve the following Cauchy problem for ordinary differential equations

$$
\begin{aligned}
& \frac{\mathrm{d}}{\mathrm{d} t}\left(\boldsymbol{w}_{m}, \boldsymbol{E}_{i}\right)+\nu\left(\nabla \boldsymbol{w}_{m}, \nabla \boldsymbol{E}_{i}\right)-\left(\boldsymbol{w}_{m} \otimes \boldsymbol{w}_{m}, \nabla \overline{\boldsymbol{E}}_{i}\right)=-\left(\rho_{m} e_{3}, \boldsymbol{E}_{i}\right), \\
& \frac{\mathrm{d}}{\mathrm{d} t}\left(\rho_{m}, E_{i}\right)+K\left(\nabla \rho_{m}, \nabla E_{i}\right)-\left(\rho_{m} \boldsymbol{w}_{m}, \nabla \bar{E}_{i}\right)=0, \\
& \boldsymbol{w}_{m}(0)=\boldsymbol{P}_{m}(\boldsymbol{w}(0)), \quad \rho_{m}(0)=P_{m}(\rho(0)),
\end{aligned}
$$

for $i=1, \ldots, m$.

Local existence follows from a basic theorem on ordinary differential equations and we look for a priori estimates on the life-span independent of $m$. For each scalar or vector valued function $v$, we define the following norm weighted with $\alpha$ (cf. the definition of the functional spaces in Sec. 2):

$$
\|v\|_{\text {vert }}^{2}:=\|v\|^{2}+\alpha^{2}\left\|\partial_{3} v\right\|^{2} .
$$

We test the first equation against $A_{3} \boldsymbol{w}_{m}$ and use the following identity (see [3] for its proof)

$$
-\left(\boldsymbol{w}_{m} \otimes \boldsymbol{w}_{m}, \nabla \overline{A_{3} \boldsymbol{w}_{m}}\right)=\left(\overline{\nabla \cdot\left(\boldsymbol{w}_{m} \otimes \boldsymbol{w}_{m}\right)}, A_{3} \boldsymbol{w}_{m}\right)=0,
$$

to get the following differential inequality:

$$
\begin{aligned}
& \frac{1}{2} \frac{\mathrm{d}}{\mathrm{d} t}\left(\left\|\boldsymbol{w}_{m}\right\|^{2}+\alpha^{2}\left\|\partial_{3} \boldsymbol{w}_{m}\right\|^{2}\right)+\nu\left(\left\|\nabla \boldsymbol{w}_{m}\right\|^{2}+\alpha^{2}\left\|\partial_{3} \nabla \boldsymbol{w}_{m}\right\|^{2}\right) \\
& \quad=-\left(\rho_{m} e_{3}, A_{3} \boldsymbol{w}_{m}\right)=-\left(\rho_{m} e_{3}, \boldsymbol{w}_{m}\right)-\alpha^{2}\left(\partial_{3} \rho_{m} e_{3}, \partial_{3} \boldsymbol{w}_{m}\right) \\
& \quad \leq \frac{1}{2}\left\|\rho_{m}\right\|^{2}+\frac{1}{2}\left\|\boldsymbol{w}_{m}\right\|^{2}+\frac{\alpha^{2}}{2}\left\|\partial_{3} \rho_{m}\right\|^{2}+\frac{\alpha^{2}}{2}\left\|\partial_{3} \boldsymbol{w}_{m}\right\|^{2} .
\end{aligned}
$$


We look for similar estimates for $\rho_{m}$ and so, analogously, we test the second equation against $A_{3} \rho_{m}$ and use

$$
-\left(\overline{\rho_{m} \boldsymbol{w}_{m}}, A_{3} \nabla \rho_{m}\right)=-\left(\rho_{m} \boldsymbol{w}_{m}, \nabla \rho_{m}\right)=0,
$$

(due to $\nabla \cdot \boldsymbol{w}_{m}=0$; note that we need to test against $A_{3} \rho_{m}$ in order to cancel the filter and obtain the previous identity) to get

$$
\frac{1}{2} \frac{\mathrm{d}}{\mathrm{d} t}\left(\left\|\rho_{m}\right\|^{2}+\alpha^{2}\left\|\partial_{3} \rho_{m}\right\|^{2}\right)+K\left(\left\|\nabla \rho_{m}\right\|^{2}+\alpha^{2}\left\|\partial_{3} \nabla \rho_{m}\right\|^{2}\right)=0 .
$$

Summing up the latest two differential inequalities, we obtain

$$
\begin{gathered}
\frac{1}{2} \frac{\mathrm{d}}{\mathrm{d} t}\left(\left\|\boldsymbol{w}_{m}\right\|_{\text {vert }}^{2}+\left\|\rho_{m}\right\|_{\text {vert }}^{2}\right)+\nu\left\|\nabla \boldsymbol{w}_{m}\right\|_{\text {vert }}^{2}+K\left\|\nabla \rho_{m}\right\|_{\text {vert }}^{2} \\
\leq \frac{1}{2}\left(\left\|\boldsymbol{w}_{m}\right\|_{\text {vert }}^{2}+\left\|\rho_{m}\right\|_{\text {vert }}^{2}\right) .
\end{gathered}
$$

An application of the Gronwall lemma gives

$$
\left\|\boldsymbol{w}_{m}(t)\right\|_{\text {vert }}^{2}+\left\|\rho_{m}(t)\right\|_{\text {vert }}^{2} \leq \mathrm{e}^{t}\left(\left\|\boldsymbol{w}_{m}(0)\right\|_{\text {vert }}^{2}+\left\|\rho_{m}(0)\right\|_{\text {vert }}^{2}\right),
$$

so that $\boldsymbol{w}_{m} \in \mathrm{L}^{\infty}\left(0, T ; \boldsymbol{V}_{\text {vert }}\right)$ and $\rho_{m} \in \mathrm{L}^{\infty}\left(0, T ; H_{\text {vert }}^{1}\right)$. Integrating (4) on $[0, T]$, we have $\boldsymbol{w}_{m} \in$ $\mathrm{L}^{2}\left(0, T ; \boldsymbol{V} \cap \boldsymbol{H}_{\text {vert }}^{2}\right)$ and $\rho_{m} \in \mathrm{L}^{2}\left(0, T ; \mathrm{H}^{1} \cap H_{\text {vert }}^{2}\right)$. Thus, proceeding as in Remark 3.2, we obtain $\nabla_{\mathrm{h}} \cdot \boldsymbol{w}_{m}^{\mathrm{h}} \in \mathrm{L}^{\infty}\left(0, T ; \mathrm{L}^{2}\right) \cap \mathrm{L}^{2}\left(0, T ; \mathrm{H}^{1}\right)$.

Now, we need estimates in order to pass to the limit as $m \rightarrow+\infty$. The quantities involved in the nonlinear terms can be estimated using the Hölder and the Gagliardo-Nirenberg inequalities. By applying Lemma 3.4, we have $\partial_{t} \boldsymbol{w}_{m} \in \mathrm{L}^{2}\left(0, T ; \boldsymbol{V}^{*}\right)$ (see [1], Step 2 in the proof of Theorem 1.1; note that our case corresponds to the deconvolution parameter $N=0$ ).

As for what concerns $\partial_{t} \rho_{m}$, we have

$$
\begin{aligned}
\left(\overline{\rho_{m} \boldsymbol{w}_{m}}, \nabla \psi\right) & =\left(\rho_{m} w_{m}^{3}, \partial_{3} \bar{\psi}\right)+\left(\rho_{m} \boldsymbol{w}_{m}^{\mathrm{h}}, \nabla_{\mathrm{h}} \bar{\psi}\right) \\
& =\left(\rho_{m} w_{m}^{3}, \partial_{3} \bar{\psi}\right)+\left(\left(\boldsymbol{w}_{m}^{\mathrm{h}} \cdot \nabla_{\mathrm{h}}\right) \bar{\psi}, \rho_{m}\right),
\end{aligned}
$$

and hence, by using the Hölder and the Gagliardo-Nirenberg inequalities for the first term, and Lemma 3.4 for the second one, we obtain

$$
\begin{aligned}
& \left|\left(\overline{\rho_{m}(t) \boldsymbol{w}_{m}(t)}, \nabla \psi(t)\right)\right| \leq\left|\left(\rho_{m}(t) w_{m}^{3}(t), \partial_{3} \bar{\psi}(t)\right)\right|+\left|\left(\left(\boldsymbol{w}_{m}^{\mathrm{h}}(t) \cdot \nabla_{\mathrm{h}}\right) \bar{\psi}(t), \rho_{m}(t)\right)\right| \\
& \leq\left\|\rho_{m}(t)\right\|_{\mathrm{L}^{2}}\left\|\boldsymbol{w}_{m}(t)\right\|_{\mathrm{L}^{3}}\left\|\partial_{3} \bar{\psi}(t)\right\|_{\mathrm{L}^{6}} \\
& \quad+C\left\|\rho_{m}(t)\right\|_{\mathrm{L}^{2}}^{1 / 2}\left\|\nabla \rho_{m}(t)\right\|_{\mathrm{L}^{2}}^{1 / 2}\left\|\boldsymbol{w}_{m}^{\mathrm{h}}(t)\right\|_{\mathrm{L}^{2}}^{1 / 2}\left\|\nabla \boldsymbol{w}_{m}^{\mathrm{h}}(t)\right\|_{\mathrm{L}^{2}}^{1 / 2}\|\nabla \bar{\psi}(t)\|_{\mathrm{L}^{2}}^{1 / 2}\left\|\nabla \partial_{3} \bar{\psi}(t)\right\|_{\mathrm{L}^{2}}^{1 / 2} \\
& \leq C\left\|\rho_{m}(t)\right\|\left\|\boldsymbol{w}_{m}(t)\right\|^{1 / 2}\left\|\nabla \boldsymbol{w}_{m}(t)\right\|^{1 / 2}\left\|\partial_{3} \nabla \bar{\psi}(t)\right\| \\
& \quad+C\left\|\rho_{m}(t)\right\|^{1 / 2}\left\|\nabla \rho_{m}(t)\right\|^{1 / 2}\left\|\boldsymbol{w}_{m}(t)\right\|^{1 / 2}\left\|\nabla \boldsymbol{w}_{m}(t)\right\|^{1 / 2}\|\nabla \psi(t)\|, \\
& \leq C\left\|\boldsymbol{w}_{m}(t)\right\|^{1 / 2}\left\|\rho_{m}(t)\right\|^{1 / 2}\left\|\boldsymbol{w}_{m}(t)\right\|_{V}^{1 / 2}\left\|\rho_{m}(t)\right\|_{\mathrm{H}^{1}}^{1 / 2}\|\nabla \psi(t)\|,
\end{aligned}
$$

so that, using again the Hölder inequality, we obtain

$$
\begin{aligned}
& \int_{0}^{T}\left|\left(\overline{\rho_{m}(t) \boldsymbol{w}_{m}(t)}, \nabla \psi(t)\right)\right| \mathrm{d} t \\
& \quad \leq C\left\|\boldsymbol{w}_{m}(t)\right\|_{\mathrm{L}^{\infty}(0, T ; H)}^{1 / 2}\left\|\rho_{m}(t)\right\|_{\mathrm{L}^{\infty} \mathrm{L}^{2}}^{1 / 2}\left\|\boldsymbol{w}_{m}(t)\right\|_{\mathrm{L}^{2}(0, T ; V)}^{1 / 2}\left\|\rho_{m}(t)\right\|_{\mathrm{L}^{2} \mathrm{H}^{1}}^{1 / 2}\|\nabla \psi(t)\|_{\mathrm{L}^{2} \mathrm{~L}^{2}} .
\end{aligned}
$$


In order to estimate $\left|\left(\nabla \rho_{m}, \nabla \psi\right)\right|$, we utilize $\rho_{m} \in \mathrm{L}^{2}\left(0, T ; \mathrm{H}^{1}\right)$ to get

$$
\int_{0}^{T}\left|\left(\nabla \rho_{m}, \nabla \psi\right)\right| \mathrm{d} t \leq\left\|\nabla \rho_{m}\right\|_{\mathrm{L}^{2} \mathrm{~L}^{2}}\|\nabla \psi\|_{\mathrm{L}^{2} \mathrm{~L}^{2}}
$$

so that $\partial_{t} \rho_{m} \in \mathrm{L}^{2}\left(0, T ; \mathrm{H}^{-1}\right)$, which holds for $K \geq 0$.

The estimates for the time derivatives, together with standard compactness results as the AubinLions lemma, imply that there exist $\boldsymbol{w}$ and $\rho$ regular as in the definition of regular weak solutions such that, up to sub-sequences,

$$
\boldsymbol{w}_{m} \rightarrow \boldsymbol{w}, \quad \rho_{m} \rightarrow \rho
$$

in $\mathrm{L}^{2}\left(0, T ; \mathbf{L}^{2}(D)\right)$, resp. $\mathrm{L}^{2}\left(0, T ; \mathrm{L}^{2}(D)\right)$, as $m \rightarrow+\infty$. This enables to pass to the limit in the nonlinear terms and to get that $(\boldsymbol{w}, \rho)$ is a regular weak solution to the model. Moreover, by using standard lower semicontinuity results, we obtain that such a solution satisfies the energy inequality

$$
\begin{aligned}
& \frac{1}{2}\left(\|\boldsymbol{w}(t)\|_{\text {vert }}^{2}+\|\rho(t)\|_{\text {vert }}^{2}\right)+\nu \int_{0}^{t}\|\nabla \boldsymbol{w}(s)\|_{\text {vert }}^{2} \mathrm{~d} s+K \int_{0}^{t}\|\nabla \rho(s)\|_{\text {vert }}^{2} \mathrm{~d} s \\
& \leq \frac{1}{2}\left(\|\boldsymbol{w}(0)\|_{\text {vert }}^{2}+\|\rho(0)\|_{\text {vert }}^{2}\right)-\int_{0}^{t}\left(\rho(s) e_{3}, \boldsymbol{w}(s)\right) \mathrm{d} s .
\end{aligned}
$$

Finally, we deduce $\boldsymbol{w} \in \mathscr{C}_{\mathrm{w}}\left(0, T ; \boldsymbol{V}_{\text {vert }}\right)$ and $\rho \in \mathscr{C}_{\mathrm{w}}\left(0, T ; H_{\text {vert }}^{1}\right)$.

\subsection{Energy identity}

Since the energy identity is not proved in [1], and only the energy inequality is stated, we give here some of the missing details to handle also this situation. Let $\boldsymbol{w}_{\varepsilon}, \rho_{\varepsilon}$ denote a standard regularization (obtained by convolution in time) of $\boldsymbol{w}, \rho$ (and so on), with $0<t_{0}<t_{1}<T$ fixed, and $0<\varepsilon<t_{0}$, $\varepsilon<T-t_{1}, \varepsilon<t_{1}-t_{0}$. For each $t \in\left[t_{0}, t_{1}\right]$, we define

$$
\boldsymbol{w}_{\varepsilon}(t)=\left(j_{\varepsilon} * \boldsymbol{w}\right)(t)=\int_{t_{0}}^{t_{1}} j_{\varepsilon}(t-s) \boldsymbol{w}(s) \mathrm{d} s,
$$

where the smooth function $j_{\varepsilon}$ is even, positive, supported in $]-\varepsilon, \varepsilon\left[\right.$, and such that $\int_{-\varepsilon}^{\varepsilon} j_{\varepsilon}(s) \mathrm{d} s=1$. Under these assumptions, we have, for any $\boldsymbol{w} \in \mathrm{L}^{q}\left(t_{0}, t_{1} ; \boldsymbol{X}\right)$ with $1 \leq q<+\infty$ and with $\boldsymbol{X}$ a Hilbert space, the following properties (see Galdi [16]).

Lemma 3.5 Under the hypotheses as above we have the following results:

1. $\boldsymbol{w}_{\varepsilon} \in \mathscr{C}^{\infty}\left(\left[t_{0}, t_{1}\right] ; \boldsymbol{X}\right)$;

2. $\lim _{\varepsilon \rightarrow 0}\left\|\boldsymbol{w}_{\varepsilon}-\boldsymbol{w}\right\|_{L^{q}\left(t_{0}, t_{1} ; \boldsymbol{X}\right)}=0$;

3. $\lim _{k \rightarrow+\infty}\left\|\left[\boldsymbol{w}_{k}\right]_{\varepsilon}-\boldsymbol{w}_{\varepsilon}\right\|_{L^{q}\left(t_{0}, t_{1} ; \boldsymbol{X}\right)}=0$ for each sequence $\left\{\boldsymbol{w}_{k}\right\} \subset \mathrm{L}^{q}\left(t_{0}, t_{1} ; \boldsymbol{X}\right)$ such that $\boldsymbol{w}_{k} \rightarrow \boldsymbol{w}$ in $\mathrm{L}^{q}\left(t_{0}, t_{1} ; \boldsymbol{X}\right)$, as $k \rightarrow+\infty$. 
Let be given two sequences

$$
\begin{array}{lll}
\boldsymbol{w}_{k} \rightarrow \boldsymbol{w} & \text { in } & \mathrm{L}^{\infty}\left(0, T ; \boldsymbol{V}_{\text {vert }}\right) \cap \mathrm{L}^{2}\left(0, T ; \boldsymbol{V} \cap \boldsymbol{H}_{\text {vert }}^{2}\right), \\
\rho_{k} \rightarrow \rho & \text { in } & \mathrm{L}^{\infty}\left(0, T ; H_{\text {vert }}^{1}\right) \cap \mathrm{L}^{2}\left(0, T ; \mathrm{H}^{1} \cap H_{\text {vert }}^{2}\right)
\end{array}
$$

we use as test functions in the weak formulation $A_{3} \boldsymbol{w}_{k, \varepsilon}$ in the first equation and $A_{3} \rho_{k, \varepsilon}$ in the second one. We obtain the following equalities:

$$
\begin{aligned}
\int_{t_{0}}^{t_{1}} & \left\{\left(\boldsymbol{w}, \partial_{t} A_{3} \boldsymbol{w}_{k, \varepsilon}\right)-\nu\left(\nabla \boldsymbol{w}, \nabla A_{3} \boldsymbol{w}_{k, \varepsilon}\right)+\left(\boldsymbol{w} \otimes \boldsymbol{w}, \nabla \boldsymbol{w}_{k, \varepsilon}\right)\right\}(s) \mathrm{d} s \\
& =\int_{t_{0}}^{t_{1}}\left(\rho e_{3}, A_{3} \boldsymbol{w}_{k, \varepsilon}\right)(s) \mathrm{d} s+\left(\boldsymbol{w}\left(t_{1}\right), A_{3} \boldsymbol{w}_{k, \varepsilon}\left(t_{1}\right)\right)-\left(\boldsymbol{w}\left(t_{0}\right), A_{3} \boldsymbol{w}_{k, \varepsilon}\left(t_{0}\right)\right), \\
\int_{t_{0}}^{t_{1}} & \left\{\left(\rho, \partial_{t} A_{3} \rho_{k, \varepsilon}\right)+\left(\rho \boldsymbol{w}, \nabla \rho_{k, \varepsilon}\right)-K\left(\nabla \rho, \nabla A_{3} \rho_{k, \varepsilon}\right)\right\}(s) \mathrm{d} s \\
& =\left(\rho\left(t_{1}\right), A_{3} \rho_{k, \varepsilon}\left(t_{1}\right)\right)-\left(\rho\left(t_{0}\right), A_{3} \rho_{k, \varepsilon}\left(t_{0}\right)\right) .
\end{aligned}
$$

Summing up the two identities and using $A_{3}=I-\alpha^{2} \partial_{3}^{2}$ yields

$$
\begin{aligned}
\int_{t_{0}}^{t_{1}} & \left\{\left(\boldsymbol{w}, \partial_{t} \boldsymbol{w}_{k, \varepsilon}\right)+\alpha^{2}\left(\partial_{3} \boldsymbol{w}, \partial_{t} \partial_{3} \boldsymbol{w}_{k, \varepsilon}\right)-\nu\left(\nabla \boldsymbol{w}, \nabla \boldsymbol{w}_{k, \varepsilon}\right)-\nu \alpha^{2}\left(\partial_{3} \nabla \boldsymbol{w}, \partial_{3} \nabla \boldsymbol{w}_{k, \varepsilon}\right)\right. \\
& +\left((\boldsymbol{w} \cdot \nabla) \boldsymbol{w}_{k, \varepsilon}, \boldsymbol{w}\right)+\left(\rho, \partial_{t} \rho_{k, \varepsilon}\right)+\alpha^{2}\left(\partial_{3} \rho, \partial_{t} \partial_{3} \rho_{k, \varepsilon}\right)+\left(\rho \boldsymbol{w}, \nabla \rho_{k, \varepsilon}\right) \\
& \left.-K\left(\nabla \rho, \nabla \rho_{k, \varepsilon}\right)-K \alpha^{2}\left(\partial_{3} \nabla \rho, \partial_{3} \nabla \rho_{k, \varepsilon}\right)\right\}(s) \mathrm{d} s \\
= & \left(\boldsymbol{w}\left(t_{1}\right), \boldsymbol{w}_{k, \varepsilon}\left(t_{1}\right)\right)+\alpha^{2}\left(\partial_{3} \boldsymbol{w}\left(t_{1}\right), \partial_{3} \boldsymbol{w}_{k, \varepsilon}\left(t_{1}\right)\right)+\left(\rho\left(t_{1}\right), \rho_{k, \varepsilon}\left(t_{1}\right)\right)+\alpha^{2}\left(\partial_{3} \rho\left(t_{1}\right), \partial_{3} \rho_{k, \varepsilon}\left(t_{1}\right)\right) \\
& -\left(\boldsymbol{w}\left(t_{0}\right), \boldsymbol{w}_{k, \varepsilon}\left(t_{0}\right)\right)-\alpha^{2}\left(\partial_{3} \boldsymbol{w}\left(t_{0}\right), \partial_{3} \boldsymbol{w}_{k, \varepsilon}\left(t_{0}\right)\right) \\
& -\left(\rho\left(t_{0}\right), \rho_{k, \varepsilon}\left(t_{0}\right)\right)-\alpha^{2}\left(\partial_{3} \rho\left(t_{0}\right), \partial_{3} \rho_{k, \varepsilon}\left(t_{0}\right)\right)+\int_{t_{0}}^{t_{1}}\left(\rho e_{3}, A_{3} \boldsymbol{w}_{k, \varepsilon}\right)(s) \mathrm{d} s .
\end{aligned}
$$

Remark 3.6 When $K=0$, we can only show that $\rho \in \mathrm{L}^{\infty}\left(0, T ; H_{\mathrm{vert}}^{1}\right)$, and hence we have only $\rho_{k} \rightarrow \rho$ in $\mathrm{L}^{2}\left(0, T ; H_{\text {vert }}^{1}\right)$. Now, the term $\left(\rho \boldsymbol{w}, \nabla \rho_{k, \varepsilon}\right)$ is problematic, since we would need the convergence property $\rho_{k} \rightarrow \rho$ in $\mathrm{L}^{2}\left(0, T ; \mathrm{H}^{1} \cap H_{\text {vert }}^{2}\right)$ in order to prove that

$$
\int_{t_{0}}^{t_{1}}\left(\rho \boldsymbol{w}, \nabla \rho_{k, \varepsilon}\right) \mathrm{d} s \rightarrow \int_{t_{0}}^{t_{1}}\left(\rho \boldsymbol{w}, \nabla \rho_{\varepsilon}\right) \mathrm{d} s,
$$

as $k \rightarrow+\infty$. This is a further reason to assume $K>0$.

First, we prove that

$$
\lim _{k \rightarrow+\infty} \int_{t_{0}}^{t_{1}}\left((\boldsymbol{w} \cdot \nabla) \boldsymbol{w}_{k, \varepsilon}, \boldsymbol{w}\right)(s) \mathrm{d} s=\int_{t_{0}}^{t_{1}}\left((\boldsymbol{w} \cdot \nabla) \boldsymbol{w}_{\varepsilon}, \boldsymbol{w}\right)(s) \mathrm{d} s .
$$

If we set $\boldsymbol{\eta}_{k}=\boldsymbol{w}_{k, \varepsilon}-\boldsymbol{w}_{\varepsilon}$, by using Lemma 3.4 we obtain

$$
\left|\left((\boldsymbol{w} \cdot \nabla) \boldsymbol{\eta}_{k}, \boldsymbol{w}\right)\right| \leq C\|\boldsymbol{w}\|\|\nabla \boldsymbol{w}\|\left\|\nabla \boldsymbol{\eta}_{k}\right\|^{1 / 2}\left\|\partial_{3} \nabla \boldsymbol{\eta}_{k}\right\|^{1 / 2}
$$


and hence

$$
\int_{0}^{T}\left|\left((\boldsymbol{w} \cdot \nabla) \boldsymbol{\eta}_{k}, \boldsymbol{w}\right)(t)\right| \mathrm{d} t \leq C\|\boldsymbol{w}\|_{\mathrm{L}^{\infty}(0, T ; \boldsymbol{H})}\|\boldsymbol{w}\|_{\mathrm{L}^{2}(0, T ; \boldsymbol{V})}\left\|\boldsymbol{\eta}_{k}\right\|_{\mathrm{L}^{2}(0, T ; \boldsymbol{V})}^{1 / 2}\left\|\boldsymbol{\eta}_{k}\right\|_{\mathrm{L}^{2}\left(0, T ; \boldsymbol{H}_{\text {vert }}^{2}\right)}^{1 / 2} .
$$

If we recall the definition of $\boldsymbol{\eta}_{k}$, and use (5) and Lemma 3.5 to deduce

$$
\left\|\boldsymbol{\eta}_{k}\right\|_{\mathrm{L}^{2}(0, T ; \boldsymbol{V})}^{1 / 2} \rightarrow 0, \quad\left\|\boldsymbol{\eta}_{k}\right\|_{\mathrm{L}^{2}\left(0, T ; \boldsymbol{H}_{\text {vert }}^{2}\right)}^{1 / 2} \rightarrow 0
$$

as $k \rightarrow+\infty$, we conclude that (7) holds.

Now, we show that

$$
\lim _{k \rightarrow+\infty} \int_{t_{0}}^{t_{1}}\left(\rho \boldsymbol{w}, \nabla \rho_{k, \varepsilon}\right)(s) \mathrm{d} s=\int_{t_{0}}^{t_{1}}\left(\rho \boldsymbol{w}, \nabla \rho_{\varepsilon}\right)(s) \mathrm{d} s .
$$

If we set $\vartheta_{k}=\rho_{k, \varepsilon}-\rho_{\varepsilon}$, by using Lemma 3.4 we have

$$
\begin{aligned}
& \left|\left(\rho \boldsymbol{w}, \nabla\left(\rho_{k, \varepsilon}-\rho_{\varepsilon}\right)\right)\right|=\left|\left((\boldsymbol{w} \cdot \nabla) \vartheta_{k}, \rho\right)\right| \\
& \quad \leq C\|\boldsymbol{w}\|^{1 / 2}\|\nabla \boldsymbol{w}\|^{1 / 2}\|\rho\|^{1 / 2}\|\nabla \rho\|^{1 / 2}\left\|\nabla \vartheta_{k}\right\|^{1 / 2}\left\|\partial_{3} \nabla \vartheta_{k}\right\|^{1 / 2}
\end{aligned}
$$

for each $s \in\left[t_{0}, t_{1}\right]$, whence

$$
\begin{aligned}
& \int_{t_{0}}^{t_{1}}\left|\left(\rho \boldsymbol{w}, \nabla\left(\rho_{k, \varepsilon}-\rho_{\varepsilon}\right)\right)(s)\right| \mathrm{d} s \\
& \left.\quad \leq C\|\boldsymbol{w}\|_{\mathrm{L}^{\infty}(0, T ; \boldsymbol{H})}^{1 / 2}\|\boldsymbol{w}\|_{\mathrm{L}^{2}(0, T ; \boldsymbol{V})}^{1 / 2}\|\rho\|_{L^{\infty}\left(0, T ; \mathrm{L}^{2}\right)}^{1 / 2}\|\rho\|_{\mathrm{L}^{2}\left(0, T ; \mathrm{H}^{1}\right)}^{1 / 2}\left\|\vartheta_{k}\right\|_{\mathrm{L}^{2}\left(0, T ; \mathrm{H}^{1}\right)}^{1 / 2}\left\|\vartheta_{k}\right\|_{\mathrm{L}^{2}\left(0, T ; H_{\mathrm{vert}}\right.}^{1 / 2}\right)
\end{aligned}
$$

Since, by the definition of $\vartheta_{k}$ and the convergence properties in (6) and in Lemma 3.5, we have $\vartheta_{k} \rightarrow 0$ in $\mathrm{L}^{\infty}\left(0, T ; H_{\text {vert }}^{1}\right) \cap \mathrm{L}^{2}\left(0, T ; \mathrm{H}^{1} \cap H_{\text {vert }}^{2}\right)$ as $k \rightarrow+\infty$, the previous estimate implies (8).

By using (7) and (8), and noticing that the other terms do not give any problem (since they are linear terms and we have convergence in appropriate spaces), by taking $k \rightarrow+\infty$, we get

$$
\begin{aligned}
\int_{t_{0}}^{t_{1}} & \left\{\left(\boldsymbol{w}, \partial_{t} \boldsymbol{w}_{\varepsilon}\right)+\alpha^{2}\left(\partial_{3} \boldsymbol{w}, \partial_{t} \partial_{3} \boldsymbol{w}_{\varepsilon}\right)-\nu\left(\nabla \boldsymbol{w}, \nabla \boldsymbol{w}_{\varepsilon}\right)-\nu \alpha^{2}\left(\partial_{3} \nabla \boldsymbol{w}, \partial_{3} \nabla \boldsymbol{w}_{\varepsilon}\right)\right. \\
& +\left((\boldsymbol{w} \cdot \nabla) \boldsymbol{w}_{\varepsilon}, \boldsymbol{w}\right)+\left(\rho, \partial_{t} \rho_{\varepsilon}\right)+\alpha^{2}\left(\partial_{3} \rho, \partial_{t} \partial_{3} \rho_{\varepsilon}\right)+\left(\rho \boldsymbol{w}, \nabla \rho_{\varepsilon}\right) \\
& \left.-K\left(\nabla \rho, \nabla \rho_{\varepsilon}\right)-K \alpha^{2}\left(\partial_{3} \nabla \rho, \partial_{3} \nabla \rho_{\varepsilon}\right)\right\}(s) \mathrm{d} s \\
=(\boldsymbol{w} & \left.\left(t_{1}\right), \boldsymbol{w}_{\varepsilon}\left(t_{1}\right)\right)+\alpha^{2}\left(\partial_{3} \boldsymbol{w}\left(t_{1}\right), \partial_{3} \boldsymbol{w}_{\varepsilon}\left(t_{1}\right)\right)+\left(\rho\left(t_{1}\right), \rho_{\varepsilon}\left(t_{1}\right)\right)+\alpha^{2}\left(\partial_{3} \rho\left(t_{1}\right), \partial_{3} \rho_{\varepsilon}\left(t_{1}\right)\right) \\
& -\left(\boldsymbol{w}\left(t_{0}\right), \boldsymbol{w}_{\varepsilon}\left(t_{0}\right)\right)-\alpha^{2}\left(\partial_{3} \boldsymbol{w}\left(t_{0}\right), \partial_{3} \boldsymbol{w}_{\varepsilon}\left(t_{0}\right)\right) \\
& -\left(\rho\left(t_{0}\right), \rho_{\varepsilon}\left(t_{0}\right)\right)-\alpha^{2}\left(\partial_{3} \rho\left(t_{0}\right), \partial_{3} \rho_{\varepsilon}\left(t_{0}\right)\right)+\int_{t_{0}}^{t_{1}}\left(\rho e_{3}, A_{3} \boldsymbol{w}_{\varepsilon}\right)(s) \mathrm{d} s .
\end{aligned}
$$

Proceeding as in [4], we can show that

$$
\begin{aligned}
& \int_{t_{0}}^{t_{1}}\left\{\left(\boldsymbol{w}, \partial_{t} \boldsymbol{w}_{\varepsilon}\right)+\alpha^{2}\left(\partial_{3} \boldsymbol{w}, \partial_{t} \partial_{3} \boldsymbol{w}_{\varepsilon}\right)+\left(\rho, \partial_{t} \rho_{\varepsilon}\right)+\alpha^{2}\left(\partial_{3} \rho, \partial_{t} \partial_{3} \rho_{\varepsilon}\right)\right\}(s) \mathrm{d} s=0, \\
& \lim _{\varepsilon \rightarrow 0} \int_{t_{0}}^{t_{1}}\left\{\left((\boldsymbol{w} \cdot \nabla) \boldsymbol{w}_{\varepsilon}, \boldsymbol{w}\right)+\left(\rho \boldsymbol{w}, \nabla \rho_{\varepsilon}\right)\right\}(s) \mathrm{d} s=0,
\end{aligned}
$$


and that the other terms converge to their natural counterparts with no $\varepsilon$. For the sake of completeness, we recall the main techniques. First, let us observe that $j_{\varepsilon}$ is supported in $]-\varepsilon, \varepsilon[$ and even, so that its derivative $j_{\varepsilon}^{\prime}$ is odd. Recalling the definition of $\boldsymbol{w}_{\varepsilon}$, we deduce

$$
\begin{gathered}
\int_{t_{0}}^{t_{1}}\left(\boldsymbol{w}(s), \partial_{t} \boldsymbol{w}_{\varepsilon}(s)\right) \mathrm{d} s=\int_{t_{0}}^{t_{1}} \int_{t_{0}}^{t_{1}} j_{\varepsilon}^{\prime}(s-r)(\boldsymbol{w}(s), \boldsymbol{w}(r)) \mathrm{d} s \mathrm{~d} r \\
=\iint_{E_{1}}+\iint_{E_{2}} j_{\varepsilon}^{\prime}(s-r)(\boldsymbol{w}(s), \boldsymbol{w}(r)) \mathrm{d} s \mathrm{~d} r=0,
\end{gathered}
$$

where

$$
\begin{aligned}
& E_{1}=\left\{(r, s) \in\left[t_{0}, t_{1}\right] \times\left[t_{0}, t_{1}\right]: r \leq s \leq r+\varepsilon\right\}, \\
& E_{2}=\left\{(r, s) \in\left[t_{0}, t_{1}\right] \times\left[t_{0}, t_{1}\right]: r-\varepsilon \leq s \leq r, r \leq t_{1}\right\} .
\end{aligned}
$$

Indeed, note that $E_{1}$ is symmetric to $E_{2}$ with respect to $s=r$, and $j_{\varepsilon}^{\prime}(s-r)$ is odd with respect to $s-r$, hence $\iint_{E_{2}}=-\iint_{E_{1}}$. Similarly, we deal with the remaining terms in (10).

Next, we prove that $\left(\boldsymbol{w}\left(t_{1}\right), \boldsymbol{w}_{\varepsilon}\left(t_{1}\right)\right)=\frac{1}{2}\left\|\boldsymbol{w}\left(t_{1}\right)\right\|^{2}+O(\varepsilon)$; similar terms (without further integrals) can be handled in the same way. First, using the fact that $j_{\varepsilon}$ is even and then performing the parameter change $r=s-t_{1}$, we have that

$$
\begin{aligned}
\boldsymbol{w}_{\varepsilon}\left(t_{1}\right) & =\int_{t_{0}}^{t_{1}} j_{\varepsilon}\left(t_{1}-s\right) \boldsymbol{w}(s) \mathrm{d} s \\
& =\int_{t_{0}-t_{1}}^{0} j_{\varepsilon}(r) \boldsymbol{w}\left(r+t_{1}\right) \mathrm{d} r=\int_{-\varepsilon}^{0} j_{\varepsilon}(r) \boldsymbol{w}\left(r+t_{1}\right) \mathrm{d} r,
\end{aligned}
$$

since $j_{\varepsilon}$ is supported in $]-\varepsilon, \varepsilon\left[\right.$ and $t_{0}-t_{1}<-\varepsilon$. Thus

$$
\begin{aligned}
& \left(\boldsymbol{w}\left(t_{1}\right), \boldsymbol{w}_{\varepsilon}\left(t_{1}\right)\right)=\int_{-\varepsilon}^{0} j_{\varepsilon}(r)\left(\boldsymbol{w}\left(t_{1}\right), \boldsymbol{w}\left(r+t_{1}\right)\right) \mathrm{d} r \\
& \quad=\int_{-\varepsilon}^{0} j_{\varepsilon}(r)\left(\boldsymbol{w}\left(t_{1}\right), \boldsymbol{w}\left(t_{1}\right)\right) \mathrm{d} r+\int_{-\varepsilon}^{0} j_{\varepsilon}(r)\left(\boldsymbol{w}\left(t_{1}\right), \boldsymbol{w}\left(r+t_{1}\right)-\boldsymbol{w}\left(t_{1}\right)\right) \mathrm{d} r \\
& =\frac{1}{2}\left\|\boldsymbol{w}\left(t_{1}\right)\right\|^{2}+O(\varepsilon),
\end{aligned}
$$

where we have used $\int_{-\varepsilon}^{0} j_{\varepsilon}(r) \mathrm{d} r=\frac{1}{2}$ for the first term and $\boldsymbol{w} \in \mathscr{C}_{\mathrm{w}}\left(0, T ; V_{\mathrm{h}}\right)$ for the second one.

The terms in (11), and the remaining integral terms, follow from the regularity of $\boldsymbol{w}$ and $\rho$. For instance,

$$
\begin{aligned}
\lim _{\varepsilon \rightarrow 0} \int_{t_{0}}^{t_{1}}\left\{\left((\boldsymbol{w} \cdot \nabla) \boldsymbol{w}_{\varepsilon}, \boldsymbol{w}\right)+\left(\rho \boldsymbol{w}, \nabla \rho_{\varepsilon}\right)\right\}(s) \mathrm{d} s \\
\quad=\int_{t_{0}}^{t_{1}}\{((\boldsymbol{w} \cdot \nabla) \boldsymbol{w}, \boldsymbol{w})+(\rho \boldsymbol{w}, \nabla \rho)\}(s) \mathrm{d} s=0,
\end{aligned}
$$

where the fact that the latter integral vanishes is obtained in a standard way by approximating $\boldsymbol{w}, \rho$ through smooth functions and using the fact that $\nabla \cdot \boldsymbol{w}=0$. 
Thus, taking the limit $\varepsilon \rightarrow 0$, we get the energy identity for the model:

$$
\begin{aligned}
& \frac{1}{2}\left\{\left\|\boldsymbol{w}\left(t_{1}\right)\right\|^{2}+\alpha^{2}\left\|\partial_{3} \boldsymbol{w}\left(t_{1}\right)\right\|^{2}+\left\|\rho\left(t_{1}\right)\right\|^{2}+\alpha^{2}\left\|\partial_{3} \rho\left(t_{1}\right)\right\|^{2}\right\} \\
& \quad+\int_{t_{0}}^{t_{1}}\left\{\nu\|\nabla \boldsymbol{w}(s)\|^{2}+\nu \alpha^{2}\left\|\partial_{3} \nabla \boldsymbol{w}(s)\right\|^{2}+K\|\nabla \rho(s)\|^{2}+K \alpha^{2}\left\|\partial_{3} \nabla \rho(s)\right\|^{2}\right\} \mathrm{d} s \\
& =\frac{1}{2}\left\{\left\|\boldsymbol{w}\left(t_{0}\right)\right\|^{2}+\alpha^{2}\left\|\partial_{3} \boldsymbol{w}\left(t_{0}\right)\right\|^{2}+\left\|\rho\left(t_{0}\right)\right\|^{2}+\alpha^{2}\left\|\partial_{3} \rho\left(t_{0}\right)\right\|^{2}\right\}-\int_{t_{0}}^{t_{1}}\left(\rho(s) e_{3}, A_{3} \boldsymbol{w}(s)\right) \mathrm{d} s ;
\end{aligned}
$$

taking $t_{0} \rightarrow 0$ and $t_{1}=t$, thanks to the weak continuity of $\boldsymbol{w}, \rho$, we can conclude that

$$
\begin{aligned}
& \frac{1}{2}\left\{\|\boldsymbol{w}(t)\|^{2}+\alpha^{2}\left\|\partial_{3} \boldsymbol{w}(t)\right\|^{2}+\|\rho(t)\|^{2}+\alpha^{2}\left\|\partial_{3} \rho(t)\right\|^{2}\right\} \\
& \quad+\int_{0}^{t}\left\{\nu\|\nabla \boldsymbol{w}(s)\|^{2}+\nu \alpha^{2}\left\|\partial_{3} \nabla \boldsymbol{w}(s)\right\|^{2}+K\|\nabla \rho(s)\|^{2}+K \alpha^{2}\left\|\partial_{3} \nabla \rho(s)\right\|^{2}\right\} \mathrm{d} s \\
& =\frac{1}{2}\left\{\|\boldsymbol{w}(0)\|^{2}+\alpha^{2}\left\|\partial_{3} \boldsymbol{w}(0)\right\|^{2}+\|\rho(0)\|^{2}+\alpha^{2}\left\|\partial_{3} \rho(0)\right\|^{2}\right\}-\int_{0}^{t}\left(\rho(s) e_{3}, A_{3} \boldsymbol{w}(s)\right) \mathrm{d} s,
\end{aligned}
$$

or with a more compact notation,

$$
\begin{aligned}
& \frac{1}{2}\left\{\|\boldsymbol{w}(t)\|_{\text {vert }}^{2}+\|\rho(t)\|_{\text {vert }}^{2}\right\}+\int_{0}^{t}\left\{\nu\|\nabla \boldsymbol{w}(s)\|_{\text {vert }}^{2}+K\|\nabla \rho(s)\|_{\text {vert }}^{2}\right\} \mathrm{d} s \\
& =\frac{1}{2}\left\{\|\boldsymbol{w}(0)\|_{\text {vert }}^{2}+\|\rho(0)\|_{\text {vert }}^{2}\right\}-\int_{0}^{t}\left(\rho(s) e_{3}, A_{3} \boldsymbol{w}(s)\right) \mathrm{d} s .
\end{aligned}
$$

\subsection{Uniqueness and continuous dependence}

In this section we prove the uniqueness of the regular weak solution and since the proof follows the same lines of results in $[1,4]$ we just provide the main steps. The interested reader can find further details in the cited references.

We begin by taking two regular weak solutions $\left(\boldsymbol{w}_{1}, \rho_{1}\right)$ and $\left(\boldsymbol{w}_{2}, \rho_{2}\right)$ to $(1)-(2)-(3)$ with the same initial data $\left(\boldsymbol{w}_{1}(0), \rho_{1}(0)\right)=\left(\boldsymbol{w}_{2}(0), \rho_{2}(0)\right)$. We set $\boldsymbol{w}=\boldsymbol{w}_{1}-\boldsymbol{w}_{2}$ and $\rho=\rho_{1}-\rho_{2}$, so that $(\boldsymbol{w}(0), \rho(0))=(\mathbf{0}, 0)$. We test the equations for $\partial_{t} \boldsymbol{w}_{1}$ and $\partial_{t} \boldsymbol{w}_{2}$ against $\left[A_{3} \boldsymbol{w}\right]_{k, \varepsilon}$ and the equations for $\partial_{t} \rho_{1}$ and $\partial_{t} \rho_{2}$ against $\left[A_{3} \rho\right]_{k, \varepsilon}$ (note that $A_{3} \boldsymbol{w}$ and $A_{3} \rho$ are not allowed to be used directly as test functions). Observe that the $\varepsilon$ regularization commutes with the filter $A_{3}$ and more generally with space derivatives. Using the same arguments as in the previous subsection, we can pass to the limit as $k \rightarrow+\infty$. If we subtract the equation for $\boldsymbol{w}_{2}$ from that for $\boldsymbol{w}_{1}$, we can deduce

$$
\begin{aligned}
& \int_{0}^{t}\left\{\left(\boldsymbol{w}, \partial_{t} \boldsymbol{w}_{\varepsilon}\right)+\alpha^{2}\left(\partial_{3} \boldsymbol{w}, \partial_{t} \partial_{3} \boldsymbol{w}_{\varepsilon}\right)-\nu\left(\nabla \boldsymbol{w}, \nabla \boldsymbol{w}_{\varepsilon}\right)-\nu \alpha^{2}\left(\partial_{3} \nabla \boldsymbol{w}, \partial_{3} \nabla \boldsymbol{w}_{\varepsilon}\right)\right. \\
& \left.\quad+\left(\left(\boldsymbol{w}_{1} \cdot \nabla\right) \boldsymbol{w}_{\varepsilon}, \boldsymbol{w}_{1}\right)-\left(\left(\boldsymbol{w}_{2} \cdot \nabla\right) \boldsymbol{w}_{\varepsilon}, \boldsymbol{w}_{2}\right)\right\}(s) \mathrm{d} s \\
& =\int_{0}^{t}\left(\rho e_{3}, A_{3} \boldsymbol{w}_{\varepsilon}\right)(s) \mathrm{d} s+\left(\boldsymbol{w}(t), \boldsymbol{w}_{\varepsilon}(t)\right)+\alpha^{2}\left(\partial_{3} \boldsymbol{w}(t), \partial_{3} \boldsymbol{w}_{\varepsilon}(t)\right) \\
& \quad-\left(\boldsymbol{w}(0), \boldsymbol{w}_{\varepsilon}(0)\right)-\alpha^{2}\left(\partial_{3} \boldsymbol{w}(0), \partial_{3} \boldsymbol{w}_{\varepsilon}(0)\right)
\end{aligned}
$$


since $\boldsymbol{w}(0)=\mathbf{0}$ and

$$
\begin{aligned}
& \left(\left(\boldsymbol{w}_{1} \cdot \nabla\right) \boldsymbol{w}_{\varepsilon}, \boldsymbol{w}_{1}\right)-\left(\left(\boldsymbol{w}_{2} \cdot \nabla\right) \boldsymbol{w}_{\varepsilon}, \boldsymbol{w}_{2}\right) \\
& \quad=\left((\boldsymbol{w} \cdot \nabla) \boldsymbol{w}_{\varepsilon}, \boldsymbol{w}\right)+\left(\left(\boldsymbol{w}_{2} \cdot \nabla\right) \boldsymbol{w}_{\varepsilon}, \boldsymbol{w}\right)+\left((\boldsymbol{w} \cdot \nabla) \boldsymbol{w}_{\varepsilon}, \boldsymbol{w}_{2}\right),
\end{aligned}
$$

we obtain

$$
\begin{gathered}
\int_{0}^{t}\left\{\left(\boldsymbol{w}, \partial_{t} \boldsymbol{w}_{\varepsilon}\right)+\alpha^{2}\left(\partial_{3} \boldsymbol{w}, \partial_{t} \partial_{3} \boldsymbol{w}_{\varepsilon}\right)-\nu\left(\nabla \boldsymbol{w}, \nabla \boldsymbol{w}_{\varepsilon}\right)-\nu \alpha^{2}\left(\partial_{3} \nabla \boldsymbol{w}, \partial_{3} \nabla \boldsymbol{w}_{\varepsilon}\right)\right. \\
\left.+\left((\boldsymbol{w} \cdot \nabla) \boldsymbol{w}_{\varepsilon}, \boldsymbol{w}\right)+\left(\left(\boldsymbol{w}_{2} \cdot \nabla\right) \boldsymbol{w}_{\varepsilon}, \boldsymbol{w}\right)+\left((\boldsymbol{w} \cdot \nabla) \boldsymbol{w}_{\varepsilon}, \boldsymbol{w}_{2}\right)\right\}(s) \mathrm{d} s \\
=\int_{0}^{t}\left(\rho e_{3}, A_{3} \boldsymbol{w}_{\varepsilon}\right)(s) \mathrm{d} s+\left(\boldsymbol{w}(t), \boldsymbol{w}_{\varepsilon}(t)\right)+\alpha^{2}\left(\partial_{3} \boldsymbol{w}(t), \partial_{3} \boldsymbol{w}_{\varepsilon}(t)\right) .
\end{gathered}
$$

Similarly, by subtracting the equations for $\rho_{1}$ and $\rho_{2}$, we have

$$
\begin{aligned}
& \int_{0}^{t}\left\{\left(\rho, \partial_{t} A_{3} \rho_{\varepsilon}\right)+\left(\rho_{1} \boldsymbol{w}_{1}, \nabla \rho_{\varepsilon}\right)-\left(\rho_{2} \boldsymbol{w}_{2}, \nabla \rho_{\varepsilon}\right)-K\left(\nabla \rho, \nabla A_{3} \rho_{\varepsilon}\right)\right\}(s) \mathrm{d} s \\
& \quad=\left(\rho(t), A_{3} \rho_{\varepsilon}(t)\right)-\left(\rho(0), A_{3} \rho_{\varepsilon}(0)\right) .
\end{aligned}
$$

Recalling that $\rho(0)=0$, by using

$$
\left(\rho_{1} \boldsymbol{w}_{1}, \nabla \rho_{\varepsilon}\right)-\left(\rho_{2} \boldsymbol{w}_{2}, \nabla \rho_{\varepsilon}\right)=\left(\rho \boldsymbol{w}, \nabla \rho_{\varepsilon}\right)+\left(\rho \boldsymbol{w}_{2}, \nabla \rho_{\varepsilon}\right)+\left(\rho_{2} \boldsymbol{w}, \nabla \rho_{\varepsilon}\right),
$$

and by writing the explicit expression for $A_{3}$, we obtain

$$
\begin{aligned}
& \int_{0}^{t}\left\{\left(\rho, \partial_{t} \rho_{\varepsilon}\right)+\alpha^{2}\left(\partial_{3} \rho, \partial_{t} \partial_{3} \rho_{\varepsilon}\right)+\left(\rho \boldsymbol{w}, \nabla \rho_{\varepsilon}\right)+\left(\rho \boldsymbol{w}_{2}, \nabla \rho_{\varepsilon}\right)+\left(\rho_{2} \boldsymbol{w}, \nabla \rho_{\varepsilon}\right)\right. \\
& \left.\quad-K\left(\nabla \rho, \nabla \rho_{\varepsilon}\right)-K \alpha^{2}\left(\partial_{3} \nabla \rho, \partial_{3} \nabla \rho_{\varepsilon}\right)\right\}(s) \mathrm{d} s \\
& =\left(\rho(t), \rho_{\varepsilon}(t)\right)+\alpha^{2}\left(\partial_{3} \rho(t), \partial_{3} \rho_{\varepsilon}(t)\right) .
\end{aligned}
$$

If we sum (12) and (13), and take the limit as $\varepsilon \rightarrow 0$ (by proceeding as in the previous subsection, we can consider the integral over $\left[t_{0}, t_{1}\right]$ and then take $\left.t_{0} \rightarrow 0, t_{1}=t\right)$, we get

$$
\begin{aligned}
& -\nu \int_{0}^{t}\left\{\|\nabla \boldsymbol{w}(s)\|^{2}+\alpha^{2}\left\|\partial_{3} \nabla \boldsymbol{w}(s)\right\|^{2}\right\} \mathrm{d} s-K \int_{0}^{t}\left\{\|\nabla \rho(s)\|^{2}+\alpha^{2}\left\|\partial_{3} \nabla \rho(s)\right\|^{2}\right\} \mathrm{d} s \\
& +\int_{0}^{t}\left\{\left((\boldsymbol{w} \cdot \nabla) \boldsymbol{w}, \boldsymbol{w}_{2}\right)+\left(\rho_{2} \boldsymbol{w}, \nabla \rho\right)\right\}(s) \mathrm{d} s \\
& =\int_{0}^{t}\left(\rho(s) e_{3}, A_{3} \boldsymbol{w}(s)\right) \mathrm{d} s+\frac{1}{2}\left\{\|\boldsymbol{w}(t)\|^{2}+\alpha^{2}\left\|\partial_{3} \boldsymbol{w}(t)\right\|^{2}+\|\rho(t)\|^{2}+\alpha^{2}\left\|\partial_{3} \rho(t)\right\|^{2}\right\},
\end{aligned}
$$


which implies

$$
\begin{aligned}
& \frac{1}{2}\left\{\|\boldsymbol{w}(t)\|^{2}+\alpha^{2}\left\|\partial_{3} \boldsymbol{w}(t)\right\|^{2}+\|\rho(t)\|^{2}+\alpha^{2}\left\|\partial_{3} \rho(t)\right\|^{2}\right\} \\
& \quad+\nu \int_{0}^{t}\left\{\|\nabla \boldsymbol{w}(s)\|^{2}+\alpha^{2}\left\|\partial_{3} \nabla \boldsymbol{w}(s)\right\|^{2}\right\} \mathrm{d} s+K \int_{0}^{t}\left\{\|\nabla \rho(s)\|^{2}+\alpha^{2}\left\|\partial_{3} \nabla \rho(s)\right\|^{2}\right\} \mathrm{d} s \\
& \leq \frac{1}{2} \int_{0}^{t}\left\{\|\boldsymbol{w}(s)\|^{2}+\alpha^{2}\left\|\partial_{3} \boldsymbol{w}(s)\right\|^{2}+\|\rho(s)\|^{2}+\alpha^{2}\left\|\partial_{3} \rho(s)\right\|^{2}\right\} \mathrm{d} s \\
& \quad+\int_{0}^{t}\left|\left((\boldsymbol{w} \cdot \nabla) \boldsymbol{w}, \boldsymbol{w}_{2}\right)+\left(\rho_{2} \boldsymbol{w}, \nabla \rho\right)\right|(s) \mathrm{d} s .
\end{aligned}
$$

The term $\left((\boldsymbol{w} \cdot \nabla) \boldsymbol{w}, \boldsymbol{w}_{2}\right)$ can be estimated using once again Lemma 3.4 and resorting to the Young inequality:

$$
\begin{gathered}
\left|\left((\boldsymbol{w} \cdot \nabla) \boldsymbol{w}, \boldsymbol{w}_{2}\right)\right| \leq C\|\boldsymbol{w}\|^{1 / 2}\|\nabla \boldsymbol{w}\|\left\|\partial_{3} \nabla \boldsymbol{w}\right\|^{1 / 2}\left\|\boldsymbol{w}_{2}\right\|^{1 / 2}\left\|\nabla \boldsymbol{w}_{2}\right\|^{1 / 2} \\
\leq \frac{\nu}{4}\|\nabla \boldsymbol{w}\|^{2}+\frac{\nu \alpha^{2}}{2}\left\|\partial_{3} \nabla \boldsymbol{w}\right\|^{2}+\frac{C}{\nu^{3} \alpha^{2}}\left\|\boldsymbol{w}_{2}\right\|^{2}\left\|\nabla \boldsymbol{w}_{2}\right\|^{2}\|\boldsymbol{w}\|^{2}
\end{gathered}
$$

whence

$$
\begin{gathered}
\int_{0}^{t}\left|\left((\boldsymbol{w}(s) \cdot \nabla) \boldsymbol{w}(s), \boldsymbol{w}_{2}(s)\right)\right| \mathrm{d} s \leq \int_{0}^{t}\left\{\frac{\nu}{4}\|\nabla \boldsymbol{w}(s)\|^{2}+\frac{\nu \alpha^{2}}{2}\left\|\partial_{3} \nabla \boldsymbol{w}(s)\right\|^{2}\right\} \mathrm{d} s \\
+\frac{C}{\nu^{3} \alpha^{2}} \int_{0}^{t}\left\{\left\|\boldsymbol{w}_{2}(s)\right\|^{2}\left\|\nabla \boldsymbol{w}_{2}(s)\right\|^{2}\right\}\|\boldsymbol{w}(s)\|^{2} \mathrm{~d} s .
\end{gathered}
$$

Similarly,

$$
\begin{aligned}
\mid\left(\rho_{2} \boldsymbol{w}\right. & , \nabla \rho)|=|\left((\boldsymbol{w} \cdot \nabla) \rho, \rho_{2}\right) \mid \\
& \leq C\|\boldsymbol{w}\|^{1 / 2}\|\nabla \boldsymbol{w}\|^{1 / 2}\left\|\rho_{2}\right\|^{1 / 2}\left\|\nabla \rho_{2}\right\|^{1 / 2}\|\nabla \rho\|^{1 / 2}\left\|\partial_{3} \nabla \rho\right\|^{1 / 2} \\
& \leq \frac{\nu}{4}\|\nabla \boldsymbol{w}\|^{2}+\frac{K}{2}\|\nabla \rho\|^{2}+\frac{K \alpha^{2}}{2}\left\|\partial_{3} \nabla \rho\right\|^{2}+\frac{C}{\nu K^{2} \alpha^{2}}\left\|\rho_{2}\right\|^{2}\left\|\nabla \rho_{2}\right\|^{2}\|\boldsymbol{w}\|^{2} .
\end{aligned}
$$

By inserting (15)-(16) in (14), and setting

$$
\begin{aligned}
& Y(t)=\|\boldsymbol{w}(t)\|_{\text {vert }}^{2}+\|\rho(t)\|_{\text {vert }}^{2}, \\
& B(s)=1+\frac{C}{\nu \alpha^{2}}\left\{\frac{1}{\nu^{2}}\left\|\boldsymbol{w}_{2}(s)\right\|^{2}\left\|\nabla \boldsymbol{w}_{2}(s)\right\|^{2}+\frac{1}{K^{2}}\left\|\rho_{2}(s)\right\|^{2}\left\|\nabla \rho_{2}(s)\right\|^{2}\right\},
\end{aligned}
$$

we obtain

$$
Y(t)+\nu \int_{0}^{t}\|\nabla \boldsymbol{w}(s)\|_{\text {vert }}^{2} \mathrm{~d} s+K \int_{0}^{t}\|\nabla \rho(s)\|_{\text {vert }}^{2} \mathrm{~d} s \leq \int_{0}^{t} B(s) Y(s) \mathrm{d} s .
$$

Since $B(s) \in \mathrm{L}^{1}(0, T)$, an application of the Gronwall lemma implies $Y(t) \equiv 0$, i.e. uniqueness of the solution and, by adapting the argument, continuous dependence on the data. 


\section{References}

[1] H. Ali. Approximate deconvolution model in a bounded domain with vertical regularization. J. Math. Anal. Appl. 408 (1), 355-363, 2013.

[2] H. Ali and Z. Ammari. On the Hausdorff dimension of singular sets for the Leray- $\alpha$ NavierStokes equations with fractional regularization. Dyn. Partial Differ. Equ. 9, 261-271, 2012.

[3] L.C. Berselli. Analysis of a large eddy simulation model based on anisotropic filtering. J. Math. Anal. Appl. 386, 149-170, 2012.

[4] L.C. Berselli and D. Catania. On the well-posedness of the Boussinesq equations with horizontal filter for turbulent flows. Z. Anal. Anwend. 2014, accepted for publication.

[5] L.C. Berselli, D. Catania and R. Lewandowski. Convergence of approximate deconvolution models to the mean magnetohydrodynamics equations: analysis of two models. J. Math. Anal. Appl. 401 (2), 864-880, 2013.

[6] L.C. Berselli, T. Iliescu and W.J. Layton. Mathematics of large eddy simulation of turbulent flows. Sci. Comput., Springer-Verlag, Berlin, 2006.

[7] L.C. Berselli and R. Lewandowski. Convergence of approximate deconvolution models to the mean Navier-Stokes equations. Ann. Inst. H. Poincaré Anal. Non Linéaire 29 (2), 171-198, 2012.

[8] L. Bisconti. On the convergence of an approximate deconvolution model to the $3 d$ mean Boussinesq equations. Math. Meth. Appl. Sci. 2014, accepted for publication.

[9] Y. Cao, E. M. Lunasin and E. S. Titi. Global well-posedness of the three-dimensional viscous and inviscid simplified Bardina turbulence models. Commun. Math. Sci. 4 (4), 823-848, 2006.

[10] D. Catania. Global Attractor and Determining Modes for a Hyperbolic MHD Turbulence Model. J. Turbul. 12 (40), 1-20, 2011.

[11] D. Catania. Length-scale estimates for the 3D Simplified Bardina MHD. J. Math. Phys. 52, $1-13,2011$.

[12] D. Catania. Finite dimensional global attractor for 3D MHD- $\alpha$ models: A comparison. J. Math. Fluid Mech. 14 (1), 95-115, 2012.

[13] J.-Y. Chemin, B. Desjardins, I. Gallagher and E. Grenier. Fluids with anisotropic viscosity. M2AN Math. Model. Numer. Anal. 34 (2), 315-335, 2000.

[14] J.W. Deardorff. A numerical study of three-dimensional turbulent channel flow at large Reynolds numbers. J. Fluid Mech. 41, 453-480, 1970.

[15] C. Foias, D. Holm and E.S. Titi. The three dimensional viscous Camassa-Holm equations and their relation to the Navier-Stokes equations and turbulence theory. J. Dynam. Differential Equations 14, 1-35, 2002.

[16] G.P. Galdi. An Introduction to the Navier-Stokes initial-boundary value problem. Fundamental Directions in Fluid Mechanics, Adv. Math. Fluid Mech., Birkhäuser, Basel, 1-70, 2000. 
[17] M. Germano. Differential filters for the large eddy numerical simulation of turbulent flows. Phys. Fluids 29 (6), 1755-1757, 1986.

[18] W.J. Layton and R. Lewandowski. On a well-posed turbulence model. Discrete Contin. Dyn. Syst. Ser. B 6(1), 111-128, 2006

[19] T. Özgökmen, T. Iliescu, and P. Fischer. Large eddy simulation of stratified mixing in a threedimensional lock-exchange system. Ocean Modelling 26, 134-155, 2009.

[20] M. Paicu and G. Raugel. Anisotropic Navier-Stokes equations in a bounded cylindrical domain. Partial differential equations and fluid mechanics, London Math. soc. Lecture Note Ser., 364, 146-184, 2009.

[21] B. Rummler. The eigenfunctions of the Stokes operator in special domains. I. Z. Angew. Math. Mech. 77, 619-627, 1997.

[22] S. Stolz and N. A. Adams. An approximate deconvolution procedure for large-eddy simulation. Phys. Fluids 11 (7), 1699-1701, 1999.

[23] S. Stolz, N.A. Adams and L. Kleiser. An approximate deconvolution model for large-eddy simulation with application to incompressible wall-bounded flows. Phys. Fluids 13 (4), 997$1015,2001$. 